\title{
REFEREE ACKNOWLEDGMENTS
}

All papers received and published by The Energy Journal are sent to external referees for anonymous peer review. Without the help of hundreds of expert referees, the quality and standards of the Journal could not be maintained. Below is a list of referees who have volunteered their time and talents to appraise submissions in the past year. The editors sincerely thank all referees for their efforts. Referees included as of June 1, 2007.

Tilak Abeysinghe

Philip Adams

Seabron Adamson

Bahram Adrangi

Erik Ahlgren

Jim Airola

Viren Ajodhia

Vicent Alcantara

Joseph Aldy

A.F. Alhajji

Geoffrey Allen

Dennis Anderson

Philip Andrews-Speed

Beng Wah Ang

Jay Apt

Angel Arcos

Margaret Armstrong

Robert Arnott

Pablo Arocena

Hiroshi Asano

Frank Asche

John Ashton

Izak Atiyas

Frank Atkins

Emil Attanasi

Shimon Awerbuch

Robert Ayres

Mustafa Babiker

Lance Bachmeier

Necmi Bagdadioglu

Mohsen Bahmani-Oskooee

Elizabeth Bailey

Richard Baillie

Erin Baker
National University of Singapore Centre of Policy Studies, Monash University

CRA International, Cambridge MA

University of Portland

Dept of Energy and Environment, Chalmers University

Naval Postgraduate School University of Delft

Autonomous University of Barcelona

Resources for the Future

Ohio Northern University

University of Massachusetts

Imperial College, ICCEPT, London UK

University of Dundee, CEPMLP

National University of Singapore

Carnegie Mellon University

Endesa, Spain

Ecole de Mines de Paris, CERNA

Oxford Institute for Energy Studies, UK

University of Navarra University of Tokyo

University of Stavanger

University of East Anglia, Centre for Competition \& Regulation

Sabanci Univeristy, Istanbul University of Calgary, Dept of Economics

U.S. Geological Survey

University of Sussex, UK

INSEAD, Management and the Environment MIT Joint Program on Science \& Policy of Global Change

Kansas State University

University of East Anglia

University of Wisconsin-Milwaukee

NERA Economic Consultants

Michigan State University

University of Massachusetts Amherst 
Ross Baldick

Ed Balistreri

Charles Ballard

Spencer Banzhaf

Arnold Baker

Terry Barker

Matthew Barmack

Douglas Barnes

William Barnett

Robert Bartels

Bernard Beaudreau

Michael Beenstock

Adolfo Benavides

Fred Benth

Jan Bentzen

Douglas Berg

Sandford Berg

Jean-Thomas Bernard

Paul Bernstein

Geoff Bertram

Paolo Bertoldi

John Besant-jones

Subhes Bhattacharyya

Andrea Bigano

Petter Bjerksund

David Bjornstad

Thomas B Bjorner

David Bjornstad

Odile Blanchard

Kornelis Blok

Gordon Bloomquist

Seth Blumsack

Carl Blumstein

Frederico Boffa

Christoph Bohringer

Friedel Bolle

David Bonilla

Annette Boom

Anthony Bopp

Severin Borenstein

Valentina Bosetti

Audun Botterud

Pal Boug

Alain Bousquet

Joseph Bowring

Gale Boyd

Joseph Boyer

Steven Braithwait
The University of Texas at Austin Colorado School of Mines Michigan State University Resources For The Future

Sandia National Laboratory University of Cambridge Analysis Group, Inc. San Francisco The World Bank

University of Kansas, Department of Economics University of Sydney, Economics \& Business Universite Laval, Quebec Hebrew University of Jerusalem Texas A\&M University University of Oslo Aarhus School of Business

Sam Houston State University Public Utility Research Center, Florida University Laval, Department of Economics Charles Rivers Associates Victoria University of Wellington European Commission The World Bank University of Dundee FEEM, Milan, Italty NHH, Norway

Oak Ridge National Laboratory AKF Inst. of Local Gov’t Studies Denmark

Oak Ridge National Laboratory University Pierre Mendes-France Ecofys, The Netherlands Washington State University Carnegie Mellon University UC Energy Institute, Berkeley Northerwestern University Centre for European Economic Research ZEW Europa University Viadrina, Frankfurt University of Cambridge Copenhagen Business School

James Madison University

Haas School of Business and UC Energy Institute Fondazione Eni Enrico Mattei

Argonne National Laboratory Statistics Norway

Universite de la Rochelle France PJM Interconnection

Argonne National Laboratory North Carolina State University

Christensen Associates 
Runnar Brannlund

Tim Brennan

Dagobert Brito

Stephen Brown

Gert Brunekreeft

Derek Bunn

Dallas Burtraw

James Bushnell

Torstein Arne Bye

Robert Cairns

Robin Cantor

Peter Cappers

Judith Cardell

Bjorn Carlen

Paul Carpenter

Carlo Carraro

Jennifer Castle

Steve Caudill

A. Aydin Cecen

Miguel Cerrutti

Halim Ceylan

Ujayant Chakravorty

Hing Lin Chan

Youngho Chang

Duane Chapman

Yiuhsu Chen

Martin Chick

Menzie Chinn

In-Koo Cho

Sam Choi

Aitor Ciarreta

Charlie Cicchetti

Joe Clarke

Cutler Cleveland

Tim Coelli

Matthew Cole

Timothy Considine

Frank Convery

Robert Cope

Aad Correlje

Ken Costello

Simon Cowan

Claude Crampes

Peter Cramton

Anna Creta

Patrick Criqui

Ian Cronshaw

John Cuddington
Omea University Sweden

University of Maryland Baltimore County

Rice University

Federal Reserve Bank of Dallas, TX

Tilburg University, The Netherlands

London Business School

Resources for the Future

University of California Berkeley

Statistics Norway

McGill University

Navigant Consulting

UtiliPoint International, NY

Smith College

Dept of Economics, Stockholm University

Brattle Group

University of Venice

Nuffield College, Oxford

Auburn University, Business School

Central Michigan University

State of California

Pamukkale University, Turkey

University of Central Florida

Hong Kong Baptist University

National University of Singapore

Cornell University

John Hopkins University

University of Edinburgh

University of Wisconsin

University of Illinois

University of Nevada

University of the Basque Country

University of Southern California

University of Strathclyde

Boston University

University of Queensland, Australia

University of Birmingham

The Pennsylvania State University

University College Dublin

Southeastern Louisiana University

Delft University of Technology

National Regulatory Research Institute

Oxford University

CICT France

University of Maryland

Università L. Bocconi

LEPII-EPE Grenoble, France

International Energy Agency

Colorado School of Mines 
Juncal Cunado

Daniel Czamanski

Pradeep Dadhich

Carol Dahl

Joyce Dargay

Joel Darmstader

Susmita Dasgupta

Graham A. Davis

Mary Deily

Shi-Jie Deng

Stephen DeCanio

Jeroen de Joode

Alejandro Diaz-Bautista

John Dimitropoulos

Otto Doering

Linda Doman

James Dooley

David Dorenfeld

Joseph Doucet

Stratford Douglas

Joy Dunkerley

Dominique Dupont

Mark Duvall

Issac Dyner

James S. Dyer

Robert Earle

Anton Eberhard

Andrew Eckert

Richard Eckaus

Huw Edwards

Ruud Egging

Vernon Eidman

Paul Ekins

Denny Ellerman

Caroline Elliott

Moley Espey

Bob Ethier

Joseph Eto

Bradley Ewing

Sebastian Eyre

Natalia Fabra

Barbara Farhar

Ahmad Faruqui

Frank Felder

Peter Ferderer

Chitru Fernanado

Denzil Fiebig

Frank Figge
University of Navarra

Czamanski Ben-Shahar \& Co Ltd

TERI, New Delhi, India

Colorado School of Mines

Transport Studies, University of Oxford

Resources for the Future, Washington

The World Bank

Colorado School of Mines

Lehigh University

Georgia Institute of Technology

University of California

ECN The Netherlands

Mexico

SPRU University of Sussex

Purdue University

U.S. Department of Energy

Batelle Pacific NW Labs

ExxonMobil Corp, Houston

School of Business, University of Alberta

West Virginia University

Consultant, Washington, DC

University of Twente

EPRI, Palo Alto, CA

National University of Colombia

University of Texas Austin, School of Business

Charles River Associates

University of Cape Town

Dept of Economics, University of Alberta

Massachusetts Institute of Technology

Loughborough University

ECN The Netherlands

University of Minnisota

Policy Studies Institute, UK

Massachusetts Institute of Technology

Lancaster University, Department of Economics

Clemson University

Market Monitoring, ISO New England

Lawrence Berkeley National Laboratory

Texas Tech University

John Hall Associates

Carlos III University Madrid

National Renewable Energy Laboratory

Charles River Associates, CA

Rutgers, State University of New Jersey

Macalester College

University of Oklahoma

University of NSW, School of Economics

St Andrews University 
Massimo Filippini

Dominique Finon

Carolyn Fischer

Jeffrey Fischer

Karen Fisher-Vanden

Stein-Erik Fleten

Kevin Forbes

Finn Forsund

Roger Fouquet

Mark French

Peter Fristrup

Manuel Frondel

Beatriz Gaitan-Soto

Marzio Galeotti

Edward Gallick

Paul Gallagher

Richard Garbaccio

Dermot Gately

Helyette Geman

Richard Gerlach

Reyer Gerlagh

Mike Giberson

Laurent Gilotte

Pierre Giot

Vincent Gitz

Rajeev Goel

Jose Goldemberg

Charles Goldman

Dan Goldstein

Rolf Golombek

Dan Gordon

Noriyuki Goto

Brian Gould

Fernando Gracia

Daniel Graham

Wafik Grais

Gerald Granderson

Ryan Grant

Richard Green

David Greene

Lorna Greening

Paul Gribik

James Griffin

Malcolm Grimston

Henri de Groot

Willem van Groenendaal

George Gross

Michael Grubb
University of Lugano

Energy Program of CNRS, CIRED France

Resources for the Future

Federal Trade Commission, Washington

Dartmouth College, Hanover, NH

NTNU, Norway

Catholic University of America, Washington

University of Oslo

University of the South Pacific, Fiji

Federal Reserve Board

Risoe National Laboratory

RWI, Essen, Germany

University of Hamburg

University of Milan

Federal Energy Regulatory Commission

Iowa State University

United States EPA

Dept. of Economics, New York University

Finance Dept, ESSEC, France

University of Sydney

Vrije University Amsterdam Giberson Co.

CIRED France

University of Namur, Belgium

Centre CIRED, France

Illinois State University, Dept of Economics

University of Sao Paulo, Brazil

Lawrence Berkeley National Laboratory

University of Michigan

Frischsenteret, Oslo Norway

University of Calgary

University of Tokyo, Japan

University of Wisconsin-Madison

University of Navarra, Spain

Imperial College, London UK

The World Bank

Miami University of Ohio

Analysis Group Inc

University of Birmingham, UK

Oak Ridge National Laboratory

Energy and the Environment, Los Alamos, NM

Midwest ISO

Bush School, Texas A\&M University

Royal Institute of International Affairs, London

Free University, The Netherlands

Tilburg University

University of Illinois at Urbana Champaign

TH Huxley School, Imperial College London 
Reinhard Haas

Stanton Hadley

Lester Hadsell

Catherine Hagem

Niels Haldrup

James Hamilton

Shawkat Hammoudeh

Sue Handy

Nick Hanley

Rognvaldur Hannesson

Philip Hanser

Nick Hartley

Peter Hartley

Toru Hattori

Joe Harrington

Scott Harvey

Paul Hayashi

Mark Hayes

Peter Helby

Daniel Henderson

Ana Maria Herrera

James Hewlett

Pierre Hillion

Lennart Hjalmarsson

Vladimir Hlasny

Benjamin Hobbs

William Hogan

Stephen Holland

Daniel Hollas

George Hondroyiannis

Einar Hope

Stephen Horan

Marvin Horowitz

Richard Howarth

Roy Hrab

Randy Hudson

Ronald Huisman

Benjamin Hunt

Lester Hunt

Hillard Huntington

Omowumi Iledare

Marija Ilic

Margaret Insley

Mariana Iootty

Jun Ishii

Marc Ivaldi

Imad Jabir

Christopher Jablonowski
Technical University of Vienna

Oak Ridge National Laboratory

University at Albany NY

Statistics Norway

University of Aarhus, Denmark University of California San Diego

Drexel University

University of California Davis

University of Sterling

Norwegian School of Economics and Business Admin

Brattle Group

Oxera Consulting Ltd

Rice University

CRIEPI Japan

Johns Hopkins University

Law \& Economics Consulting Group, Cambridge

University of Texas at Arlington

Stanford University

Lund University, Environmental \& Energy Systems

State University of New York, Binghamton

Michigan State University

Energy Information Administration, US DOE

INSEAD, France

Goteborg University

Michigan State University

Johns Hopkins University

Harvard University

University of North Carolina Greensboro

University of Texas San Antonio

Research Department, Bank of Greece

NHH, Norway

St. Bonaventure University

Demand Research

Environmental Studies, Dartmouth College

Ontario Energy Board

Oak Ridge National Laboratory

RSM Erasmus University, The Netherlands

International Monetary Fund

University of Surrey

Energy Modeling Forum, Stanford University

Louisiana State University

Carnegie Mellon University

University of Waterloo

Federal University of Rio de Janeiro Brazil

University of California at Irvine

CICT, France

Mount Royal College Alberta

University of Texas at Austin 
Mark Jaccard

Jonathan Jacobs

Henrick Jacobson

Amy M. Jaffe

Tooraj Jamasb

James Jensen

Tingsong Jiang

Olof Johansson

Tor Arnt Johnsen

Donald W. Jones

Clifton T. Jones

Paul L. Joskow

Frederick Joutz

Ruth Judson

Martin Junginger

Edward Kahn

Mikiko Kaimuma

Mark Kaiser

Ken Karlsson

Olaf Karstens

Robert Kaufmann

Yoichi Kaya

Lynne Keisling

Claudia Kemfert

Jussi Keppo

Neha Khanna

Daniel Khazzoom

Stephen Kidd

Lutz Killian

Brendan Kirby

Paul Kleindorfer

Andrew Kleit

Gernot Klepper

David Knapp

Christopher Knittel

Bertrand Koebel

Tina Koljonen

Jonathan Koomey

Tarjei Kristiansen

Kerry Krutilla

Onno Kuik

Timo Kuosmanen

Surender Kumar

Gurkan Kumbarloglu

Subal Kumbhakar

Ken Kurani

Gerard Kuper

Snorre Kverndokk
Simon Fraser University

PA Constulting Group, CA

Risoe National Laboratory, Denmark

Rice University, Texas

University of Cambridge

Jensen Associates Inc

Centre for International Economics, Canberra

Goteborg University, Sweden

NVE Norway

RCF Economics \& Financial Consulting

Stephen F. Austin State University

Massachusetts Institute of Technology

George Washington University

Federal Reserve Board, Washington

Utrecht University

Analysis Group/Economics

National Institute for Environmental Studies, Japan

Louisianna State University, Center for Energy Studies

RISOE National Laboratory, Denmark

Navigant Consulting Inc

Boston University

RITE, Japan

Northwestern University

Oldenburg University, Germany

University of Michigan

Cornell University

San Jose State University, California

World Nuclear Association, London

University of Michigan

Oak Ridge National Laboratory

Wharton School, University of Pennsylvania

Pennsylvania State University

IFW University of Kiel

Energy Intelligence Group

University of California at Davis

Universite Louis Pasteur

VTT Finland

Lawrence Berkeley National Laboratory

KEMA Research Consultants

University of Indiana

Vrije University, IVM, The Netherlands

MTT Economics, Agrifood Research Finland

TERI, New Delhi, India

Bogazici University, Istanbul, Turkey

State University of New York, Economics

University of California Davis

Groningen University, The Netherlands

Ragnar Frisch Centre for Economic Research 
John Kwoka

Socrates Kypreos

Xavier Labandeira

Norbert Ladoux

Stephane Lambrecht

Andreas Lange

Ian Lange

Markku Lanne

Frederic Lantz

Alessandro Lanza

Eric Larsen

Don Larson

Harri Laurikka

Delphine Lautier

Lester Lave

Steve Lawford

Chloe Le Coq

Russell Lee

Dean Leistikow

Jeff Leitzinger

Francois Lescaroux

Jonathan Lesser

Steve Letendre

Soren Leth-Petersen

Matt Lewis

Gary Libecap

Donald Lien

Jiang Lin

Pedro Linares

Tim Lipman

Wietze Lise

Matti Liski

Stephen Littlechild

Gang Liu

Asa Lofgren

Prakash Loungani

David Loughran

Knox Lovell

Mark Lowry

Tom Lundgren

Chunbo Ma

Matilde Machado

Alan MacFadyen

Gordon Mackerron

Mike Madden

Reinhard Madlener

Willy Makundi

Mike Maloney
Northeastern University

Geneva Switzerland

University of Vigo, Spain

CICT, France

University of Lille, France

University of Maryland

US Environmental Protection Agency

University of Jyvaskyla Finland

Institut Francais du Petrole

FEEM, Italy

University of Lugano

The World Bank

GreenStream Network Ltc

ENSPM France

Carnegie Mellon University

United Kingdom

University of California Berkeley

Oak Ridge National Laboratory

Fordham University

Econ One

French Petroleum Institute

Navigant Consulting/REED

Green Mountain College

AKF Denmark

University of California Energy Institute

University of Arizona

University of Texas San Antonio

Lawrence Berkeley National Laboratory

Pontifical University of Comillas Spain

University of California, Berkeley

Institute for Environmental Studies, Free University, Amsterdam

Helsinki School of Economics, Finland

Judge Institute of Management, University of Cambridge

Statistics Norway

Goteborg University

International Monetary Fund

RAND, California

The University of Georgia, College of Business

Pacific Economics Group

Swedish University of Agricultural Sciences

Rensselaer Polytechnic Institute

University Carlos III de Madrid

University of Calgary, Canada

University of Sussex

Gas Strategies Consulting Co

Swiss Federal Institutes of Technology

Lawrence Berkeley National Laboratory

Clemson University 
Matteo Manera

Erin Mansur

Jose Mantovani

James Manwell

Osmel Manzano

Gregg Marland

Chris Marnay

Joan Martinez

Isamu Matsukawa

Alex Maynard

Roger McCain

Bruce McCarl

Tanga McDaniel

Warwick McKibbin

Ross McKitrick

Robert McNown

Kenneth Medlock

Chiang Mei

Robert Mendelsohn

Tim Mennen

Sophie Meritet

Robert J. Michaels

Augusto R. Micola

Carlos de Miguel

David Mills

Catherine Mitchell

Franklin Mixon

Juan Pablo Montero

David Montgomery

Imad A. Moosa

Jim Moose

Alan Moran

Richard Morgenstern

George Moridis

Knut Anton Mork

Saeed Moshiri

Diana Moss

Timothy Mount

Edwin Muchapondwa

Erich Muehlegger

Frederic Murphy

Scott Murtishaw

Rashesh Nair

Carole Nakhle

Erkka Nasakkala

Peter Navarro

Bernie Neenan

Runa Nesbakken
University of Milano-Bicocca, Italy

School of Management, Yale University

Sao Paulo State University Brazil

Renewable Energy Research Lab, U of Mass

Corporacion Andina de Fomento

Oak Ridge National Laboratory

Lawrence Berkelely National Laboratory

Autonomous University of Barcelona

Musashi University, Japan

University of Toronto

Drexel University

Texas A\&M University, Dept Agricultural Economics

Appalachian State University

Australian National University

Dept of Economics, University of Guelph

University of Colorado, Economics Dept

Rice University, James A Baker III Institute

Massachusetts Institute of Technology

Yale University

ZEW Germany

Universite Dauphine, France

California State University, Fullerton

IMD International, Switzerland

University of Vigo Spain

University of Virginia, Economics Dept

Center Management \& Regulation, Warwick Business School

University of Southern Mississippi

Pontifical Catholic University of Chile

Charles River Associates Inc

La Trobe University, Australia

The World Bank

IPA Australia

Resources for the Future

Lawrence Berkeley National Laboratory

Handelsbanken Norway

University of Manitoba

American Antitrust Institute

Cornell Dept of Applied Economics \& Management

University of Capetown

Kennedy School, Harvard

Dept of Economics, Temple University

Lawrence Berkeley National Laboratory

National Institute for Environmental Studies, Japan

University of Surrey, UK

Helsinki University, Finland

University of California Irvine

Utilipoint

Statistics Norway 
Karsten Neuhoff

David Newbery

Anne Neumann

Shawn Xiaoguang Ni

Alessandro Nicita

David Nissen

Michael Noel

Robert Noland

Nikos Nomikos

Gbadebo Oladosu

Ole Jess Olsen

Ryota Omori

Frans Oosterhuis

Andrew Oswald

Hugh Outhred

Anthony Owen

R.K. Pachauri

Karen Palmer

Sergey Paltsev

Agis Papadopoulos

Ian Parry

Craig Parsons

Carl Pasurka

Yezid Orlando Perez Aleman

Adriaan Perrels

Frederik Petterson

Wolfgang Pfaffenberger

Andrew Pickering

Robert Pindyck

André Plourde

Steve Polasky

Michael Pollitt

Carlos Pombo

Aude Pommeret

Julia Popova

David Popp

Edward Porter

Geoff Pritchard

Stef Proost

Steven Puller

Kevin Quinn

Philip Quirion

Nuria G Rabanal

Stanislav Radchenko

Ricardo Raineri

Arvid Raknerrud

Reza Ramazani

Kevin Rask
Cambridge University, Economics

Cambridge University

Dresden University of Technology

University of Missouri

The World Bank

Columbia University, International Energy Management

University of California, San Diego

Imperial College London

Cass Business School, London

Environmental Sciences Division, ORNL

Roskilde University

National Institute of Science and Technology Policy, Japan

VU University Amsterdam

University of Warwick

University of New South Wales, Australia

Curtin University of Technology

Tata Energy Research Institute, India

Resources for the Future, Washington

Massachusetts Institute of Technology

Aristole University Thessaloniki

Resources for the Future

Yokohama National University

US Environmental Protection Agency

Pontificia University Javeriana

VATT, Finland

Lulea University

International University and Bremer Energy Institute

University of Bristol

Massachusetts Institute of Technology

University of Alberta, Canada

University of Minnisota

Judge Inst of Management, Cambridge University

University of the Andes

HEC University of Lausanne

West Virginia University

Center for Environmental Policy, Syracuse University

American Petroleum Institute

University of Auckland

Catholic University of Leuven

Texas A\&M University, Dept of Economics

St. Norbert College

Centre-CIRED France

University of Leon Spain

University of North Carolina Charlotte

Pontificia Universidad Catolica de Chile

Statistics Norway

Saint Micheal's College, Vermont

Colgate Unviersity, Economics 
Ronald Ratti

Micheal Rauscher

Dennis Ray

Hossein Razavi

Grant Read

John M. Reilly

Veronica La Regina

Stanley Reynolds

Ali M. Reza

Amy Richmond

Frank Riedel

Miguel Rodriguez

Philip Romero

Joseph Roop

Fabien Roques

Kenneth Rose

Juan Rosellon

Knut Einar Rosendahl

Christopher Ross

Michael Rothkopf

Geoffrey Rothwell

Bryan Routledge

Jonathan Rubin

Larry Ruff

Carlos Rufin

Milton Russell

Tom Rutherford

David Ryan

Perry Sadorsky

Jayant Sathaye

Vladislav Savin

Benjamin Schlesinger

Richard E. Schuler

Craig Schulman

Edwardo Schwartz

Peter Schwarz

Malte Schwoon

Frank Scrimgeour

Yadviga Semikolenova

Benoit Sevi

Deepak Sharma

Hany Shawky

Robert Shelton

Kenichi Shimamota

Mototsugu Shintani

Graham Shuttleworth

Afzal Siddiqui

Jos Sijm
University of Missouri-Columbia

Rostock University Germany

Power Systems Engineering Research Center, Madison WI

The World Bank

University of Canterbury

Massachusetts Institute of Technology

Wave Energy Centre, Lisbon

University of Arizona

San Jose State University

United States Military Academy

University of Bonn

University of Vigo, Spain

University or Oregon

Pacific Northwest National Laboratory

International Energy Agency

National Regulatory Research Institute, Ohio

CIDE Mexico

Statistics Norway

Charles River Associates Inc

Rutgers University

Stanford University

Carnegie Mellon University

University of Maine

Energy and Economic Consulting, Windsor CA

Babson College

University of Tennessee Knoxville

University of Colorado

University of Alberta

Schulich School of Business, York University

Lawrence Berkeley National Laboratory

ISEER, Russia

Benjamin Schlesinger and Associates Inc

Cornell University, College of Engineering

Law and Economics Consulting Group

Anderson School, UCLA

University of North Carolina Charlotte

GMX, Germany

University of Waikato Management School

Colorado School of Mines

University of Perpignan, France

University of Technology, Sydney

School of Business, University at Albany, NY

Oak Ridge National Laboratory

University of Birmingham, Dept of Economics

Vanderbilt University

NERA, UK

University College London

ECN, The Netherlands 
Julian Silk

Boris Silverstovs

Bernard Sinclair-Desgagne

Balbir Singh

Fereidoon Sioshansi

George Siotis

James Skea

Terje Skjerpen

Margaret Slade

Kenneth Small

James E. Smith

James L. Smith

Thomas Smith

Ron P Smith

Vaclav Smil

Lennart Soder

Patrik Soderholm

Ron Soligo

Daniel Sperling

Urs Springer

Vito Stagliano

Spiro Stefanou

Thanasis Stengos

David Stern

Jonathan Stern

Thomas Sterner

Paul Stevens

Steven Stoft

Paul Stoneman

Karl Storchmann

Eric Strobl

Francesca Stroffolini

Steinar Strom

Hiroaki Suenaga

Andrew Sweeting

Rodrigo Taborda

Richard Tabors

Michael Tamvakis

Massimo Tavoni

Beck Taylor

Jerry Taylor

Timothy Taylor

Dek Terrell

Leigh Tesfatsion

Henry Thompson

Mark Thompson

Philip Thompson

Judy Thornton
George Washington University

DIW Germany

CIRANO, HEC Montreal

SNF Institute for Research in Economics \& Business

Henwood Energy Services Inc

Universidad Carlos III de Madrid

UK Energy Research Centre

Statistics Norway

Warwick University

University of California Irvine

Fuqua School of Business, Duke University

Southern Methodist University, Texas

Zayed University, Dubai

Birkbeck College, Dept of Economics

University of Manitoba

Royal Institute of Technology, Stockholm

Lulea University of Technology, Sweden

Rice University, Texas

University of California, Davis

Ecoplan Economic Research and Policy Consultancy

Calpine Company

Pennsylvania State University

University of Guelph, Department of Economics

Rensselaer Polytechnic Institute

Oxford Institute for Energy Studies

Gothenburg Univeristy

University of Dundee, Scotland

Nantucket, MA

Warwick Business School

Whitman College, WA

Catholic University of Louvain

University of Naples

University of Oslo

Curtin University of Technology

Northwestern University, Dept of Economics

Universidad Rosario, Colombia

Charles River Associates

Cass Business School, City University London

FEEM, Italy

School of Business, Samford University

CATO Institute

University of Florida, Gainsville

Louisiana State University

Iowa State University, Dept of Economics

Auburn University

Stephen Austin University of Texas

Central Michigan University

University of Washington 
James Tipping

Jean Tirole

Asher Tishler

Tom Tietenberg

Richard Tol

Asgeir Tomasgard

Christoph Tonjes

Kenneth Train

Michael Trebilcock

Lenos Trigeorgis

Stefan Trueck

Steven Turner

Paul Twomey

Kazushi Uemura

Rocio Uria

Colin Vance

Kurt VanDender

Rick van der Ploeg

Frits Van Oostvoorn

Angel de la VegaNavarro

Shree Vikas

Laurent Viguier

Pablo Villaplana

Daniel Vine

Mahesh Vipradas

Ingo Vogelsang

Herman Vollebergh

Nils-Henrik Von der Fehr

Christian Von Hirschhausen

Laurens de Vries

Catherine Waddams

Zia Wadud

Martin Wagner

Robin Wallace

W. David Walls

Margaret Walls

Ashley Wang

Linda Warell

Mike Waterson

William Watson

Mort Webster

Jason Wei

Bernard Weinstein

Heinz Welsch

Wenlong Weng

Douglas West

Sarah West

Tom Weyman-Jones
Canterbury University

Massachusetts Institute of Technology

Tel Aviv University, Faculty of Management

Colby College

Hamburg, Vrije and Carnegie Mellon Universities

NTNU, Trondheim Norway

Clingendael Intl Energy Programme

University of California Berkeley

University of Toronto

Sloan School of Management, MIT

Queensland University of Technology

Mississippi State University

University of Cambridge

Dept Economics, Sophia University, Japan

University of California

RWI Essen Germany

University of California Irvine

IUE, Italy

ECN The Netherlands

Universidad Nacional Autonomo Mexico

SAIC Company

University of Geneva

University Pompeu Fabra of Barcelona

Federal Reserve Board

Tata Energy Research Insitute, India

Boston University, Dept of Economics

Erasmus University Rotterdam

University of Oslo, Dept of Economics

DIW, Germany

Delft University of Technology

Centre for Competition \& Regulation, Univ of East Anglia Imperial College, London

Institute for Advances Studies Vienna

University of Edinburgh

Centre for Regulatory Affairs, University of Calgary

Resources for the Future

University of California Irvine

Lulea University of Technology

University of Warwick

Energy Information Administration, US DOE

University of North Carolina

University of Toronto

University of North Texas

University of Oldenburg, Economics Dept

Lehigh University

University of Alberta

Macalester College

Loughborough University 
Halbert White

Arthur Wiese

Randy Wigle

Bert Willems

Eric Williams

Jeffrey Williams

Robert Williams

Ian Sue Wing

Harald Winkler

Joseph Winsen

Franz Wirl

Catherine Wolfram

Chi-Keung Woo

Andrew Worthington

Brian Wright

Kang Wu

Kuan Xu

Tankut Yalcinoz

Jian Yao

Adonis Yatchew

Gary Yohe

Mine Yucel

Georges Zaccour

Hailing Zang

Jay Zarnikau

Hisham Zerriffi

Yanhua Zhang

Zhen Zhu

Bob van der Zwaan
Bates White Co.

American Petroleum Institute

Wilfrid Laurier University, Waterloo, Canada Catholic University of Louvain, Belgium

Arizona State University University of California, Davis

University of Texas

Boston University, Massachusetts

Energy Research Centre, University of Capetown

University of Newcastle, Australia

University of Vienna, Austria

Haas School of Business, UC Berkeley

Energy and Environmental Economics, Inc.

University of Wollongong, Australia

University of California Berkeley

Research Program, East-West Center, Honolulu

Dalhousie University

Nigde University, Dept Electrical Engineering

UC Berkeley, IEOR

University of Toronto, Department of Economics

Wesleyan University, Economics

Federal Reserve Bank of Dallas, Texas

GERAD, Ecole des Hautes Etudes Commerciale

Texas A\&M University

Frontier Associations, Austin Texas

Stanford University

University of Toulouse

C.H. Guernsey Company

Energy Research Centre of the Netherlands 